\title{
Exploring how children interact with 3D shapes using haptic technologies
}

\author{
Nikoleta Yiannoutsou \\ Rose Johnson \\ Sara Price \\ UCL Knowledge Lab \\ UCL Institute of Education \\ University College London, \\ London, WC1N 3QS \\ n.yiannoutsou@ucl.ac.uk \\ rose.johnson.11@ucl.ac.uk \\ sara.price@ucl.ac.uk
}

Haptic devices have the potential to enhance the learning experience by foregrounding embodied, sensory and multi-modal elements of learning topics. In this paper, we report on-going work investigating a game prototype with haptic feedback for seven year old children's engagement with geometrical concepts as part of an iterative design study. Our findings include a new game play mode adopted by the children, that empowers the use of haptic feedback in game play and has the potential to enable the enactment of shape properties in the game play process.

\section{Author Keywords}

Haptic devices; children's interaction; embodied cognition

\section{ACM Classification Keywords}

H.5.2 User Interfaces: Haptic I/O; User-centered design K.3.1 Computer Uses in Education

\section{Introduction}

Permission to make digital or hard copies of all or part of this work for personal or classroom use is granted without fee provided that copies are not made or distributed for profit or commercial advantage and that copies bear this notice and the full citation on the first page. Copyrights for components of this work owned by others than ACM must be honored. Abstracting with credit is permitted. To copy otherwise, or republish, to post on servers or to redistribute to lists, requires prior specific permission and/or a fee. Request permissions from Permissions@acm.or

IDC '18, June 19-22, 2018, Trondheim, Norway (C) 2018 Association for Computing Machinery. ACM ISBN 978-1-4503-5152-2/18/06 \$15.00 https://doi.org/10.1145/3202185.3210771
The recognised importance of multi-modal and multisensory forms of interaction for learning (e.g [11]), combined with technological development, offers new opportunities for designing digital learning environments. Haptic technologies in particular offer tools for supporting tactile sensory experiences, valuable for both typically developing children and those with visual impairment. However, haptic research in elementary education contexts is limited [9], 
highlighting the need for a better understanding of young children's use (manipulation) of haptic devices, and the implications for how this might shape their reasoning. This research sits in the context of a larger project aiming to design and develop multisensory digital environments to support teaching and learning of mathematics for primary school children - both typically developing and visually impaired.

As part of an iterative design process this study investigates typically developing children's use of a haptic device to explore 2D and 3D shapes, in a purpose built serious game, designed to support geometry learning. Specifically, teacher workshops highlighted key challenges for children: understanding the third dimension of 3D shapes; recognising 3D shapes in different orientations; and the presence of the 'hidden corner', i.e. one corner of a cube cannot be seen when looking at a 3D representation of a cube [7]. Our research aims to better understand how combined tactile and visual experiences can support children's exploration of 3D figures. Specifically, this study aims to inform the game design by understanding (i) the usability or manipulation of the haptic device for young children; and (ii) ways in which the tactile experience shapes children's engagement with and interpretation of ideas of shape.

\section{Background}

Our work is situated within theories of embodied cognition, which emphasise the role of experience, the sensory body, emotion and social interaction for cognition and learning [4]. There is substantial evidence that mathematical cognition is embodied [12], being grounded in the physical environment, and based in perception and action [2]. Of key interest here is identifying the benefits of engaging with concepts through physical means, such as tactile/ haptic tools.

A key characteristic of haptic technologies is that they support manual interaction to: a) explore the environment (physical or digital) to extract information from it or/and b) manipulate the environment ([14] cited in [9]). According to Zacharia [16], haptic interaction, as exploratory tactile activity, is extended to include enhancement of perception of object or environment characteristics (e.g. shape, texture, hardness etc.) through the haptic sensation. Thus haptic technologies have the potential to add a strong tactile sensory dimension in learning. Typically, implementations consist of tactile representations combined with other form (visual, auditory, abstract). This is grounded on research showing that conceptual learning is facilitated by learners interacting with different types of information about the same concept $[1,6,9]$

Previous work using haptic devices with children has focused on those with tactile sensory loss or visually impaired, typically for rehabilitation purposes, or navigation [13]. Research with mainstream learners has primarily focused on high school science, for example: resistance between two molecules; simple machines (levers, gears, pulleys etc.); magnetic forces; mechanical forces; viruses and nanoscale science; (ibid pp. 2283). In mathematics it has been used to explore 10 year olds' interaction with a multimodal environment (haptic with 3D visuals) to support dynamic geometry learning [8] and 17 year olds' learning of trigonometry, supported by multimodal dynamic representations (abstract, visual and haptic) attaching haptic feedback to sine waves [6]. However, little work has explored 


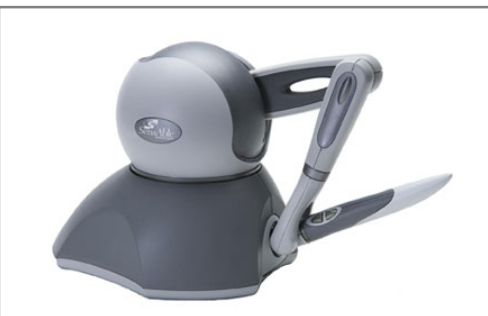

Fig. 1: Omni Phantom old generation firewire connected device

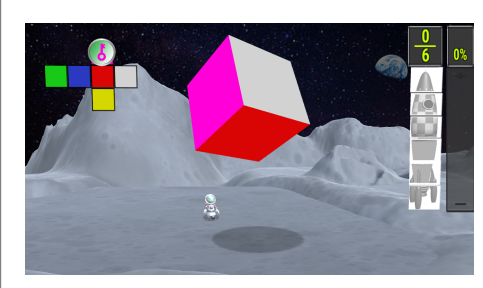

Fig. 2: Connecting the key to the cube net

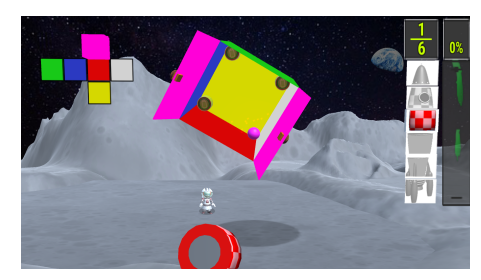

Fig 3. The first piece of the rocket and the four batteries at the vertices of the cube primary aged children's use of haptic devices.

Furthermore, current research in haptics for learning, shows inconsistent results (see $[5,16]$ ). Typically, this is due to differences in research methodology. Most studies evaluate learning using experimental methods, with control and experimental groups and pre-post testing [16]). Bival et al [5] combined quantitative and qualitative approaches. While they did not find significant differences in pre and post-tests, qualitative analysis showed that haptic interaction shaped student conception: their verbal elaborations after the interaction included tactile aspects of their sensory exploration with the haptic device. Similarly Zacharia [16] notes that further research on haptic devices for learning should look at how, when and why haptic feedback is necessary - suggesting a qualitative approach.

\section{Method}

This research is embedded in a design based research approach [15], aiming to inform design through examining how students interact and manipulate 3D space with a haptic device, and how this mediates meaning making around specific geometrical concepts.

\section{Participants and setting}

Twenty year three students (7-8 years) participated, in an in-situ constructive dyad interaction design [3], promoting children's discussion, collaboration and interaction. The study took place in a UK school during school hours with one pair of children at a time.

Session duration varied from 45 minutes to 1 hour.

\section{Data collection and analysis}

Two video cameras captured: a) children's interaction with the haptic device; b) children's interaction with the game, and c) children's discourse, including discussions with the children before and after the games. Our data analysis looked at: a) critical episodes aiming to capture breakthroughs and break-downs in student experience with the haptic feedback and b) repertoires of holding and manipulating the haptic device.

Serious Game prototype: "Space Shapes"

The serious game "Space Shapes" aims to support 3D exploration of shape through provision of physical force feedback experienced through an Omni Phantom haptic device (Fig. 1) and visual and audio feedback. In the game children explore properties of a cube (i.e. number of faces, vertices, internal corners, 2D net representation) through a narrative in which they need to find six rocket pieces to help "Mia" build her rocket. The six pieces of the rocket are accessed through the six faces of a cube. Each face has a door which opens when children connect the face to its equivalent on the cube net (using color coding) (Fig. 2). Each time the cube opens children have to manipulate the box to 'tip out' one piece of rocket. Children must also collect rocket fuel cells from each of the 4 four internal vertices (Fig.3). The next step is to assemble the rocket by putting its pieces together, one on top of the other. Force feedback is present in this prototype game, but does not play a critical role (students do not depend on it to complete the game). This study aims to investigate children's learning interactions with the haptic device to inform pedagogically driven game design.

\section{Balloon game}

The Balloon game was designed to familiarize children with the haptic device, and the perception of depth. It consists of lines of balloons situated in a 3D space. Children pop the balloons using the haptic device. 


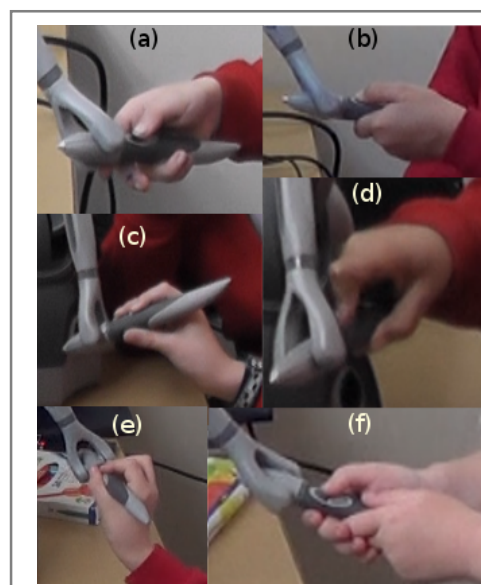

Figure 4: Variety of ways to hold the haptic device. (a) Right-hand FHTC, (b) Left-hand FHTC, (c) PHIC, (d) MHIC, (e) PHTC, (f) THTC

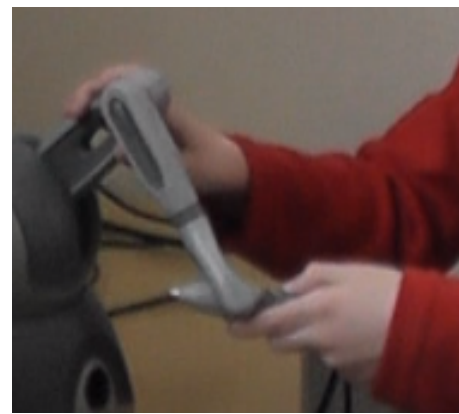

Figure 5: P10 using two hands to guide the haptic device

\section{Procedure}

Children played "Space Shapes" with the Haptic Device $(H D)$, preceded by the "Balloons" game. Before the game children answered profile questions, after the game they were asked about their experience playing. During their interaction researchers asked questions that aimed at elaboration on student comments.

\section{Student interaction with the haptic device}

We describe the preliminary findings, focusing on three key areas which have implications for design: (i) the way that the HD was held by the children, (ii) the way the HD was experienced as both a 3D input device and a source of haptic feedback, (iii) the use of haptic feedback to mediate conceptualization of shape.

\section{Holding the Haptic Device}

The HD is designed to be used like a pen. However, children did not naturally hold the device in this way, but displayed a variety of grasp styles. The most popular was to cradle the HD in four fingers and use the thumb to operate the buttons, we refer to this as Fist Hold Thumb Click (FHTC fig. 4a\&b). Eleven of the participants used this hold throughout the game and a further six participants switched to this hold by the end. Only one participant consistently used a pen hold clicking with his index finger (PHIC fig. 4c). A further four participants commenced the game using a pen hold clicking with either index or middle finger or thumb (PHTC fig. 4e) but switched to an alternative hold by the end of the game. Three participants tried a mouse-like hold with index click (MHIC fig. 4d) but none used it consistently through the game. One participant used two hands to hold the haptic device using her thumb to click the button (THTC figure 4f). Another participant who was ambidextrous changed her hold frequently through the game, at one point placing her right hand on to the HD arm for precision guidance (Fig. 5). Overall the thumb was the preferred digit for operating the button on the HD with 18 out of 20 children using their thumb for the majority of the game. In interviews students reported this to be more comfortable, with several saying it felt like an extension of their arm. Most children found it interesting to operate the HD, but also mentioned difficulties moving objects in the z-direction.

Experience of 3D Navigation and Haptic Feedback In interview, the children tended to talk most positively about the HD in terms of 3D navigation, whereas their responses to the haptic feedback were mixed. Two pairs of children (P1P2, P7P8) seemed unaware that there was haptic feedback. P7 and P8 were confused when shown it after the game saying, "it didn't really work when we done it" later concluding "we were just concentrating too much." One pair (P3P4) were aware of the haptic feedback but felt it was external control rather than tactile experience of the objects: "the device is not really, like, free. It is like someone else is grabbing the pen at the same time as I am" (P3). "[It is] like holding up a brick on the end of a paintbrush" (P4). Despite this they liked using the haptic device because it allowed them to navigate in 3D space easily.

Mediating conceptualization of $3 D$ shapes

P5 and P6 gave some indication that they perceived the haptic feedback as qualities of the objects in the game saying it felt "more rough... more like deeper" but this perception did not map to sensing 'objects in space', for when they found the haptic device prevented them from going through objects in the game they said "it doesn't work [...] it kind of makes you go round." This 


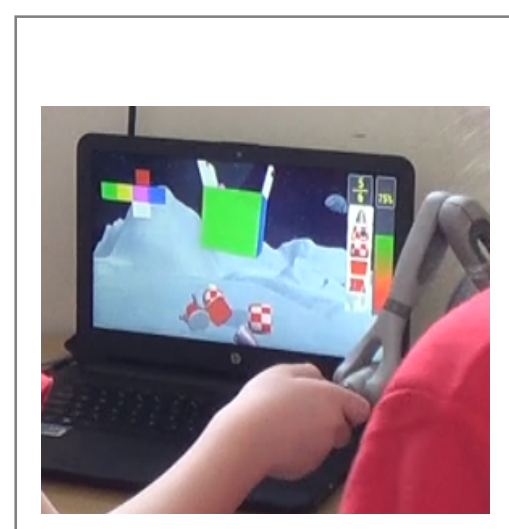

Figure 6: $\mathrm{P9}$ and $\mathrm{P} 10$ exploring the haptic feedback by turning the box upright so that they minimize the visual feedback. suggests that children may be drawing on prior gaming experience, where it is possible to move through walls, yet it also highlights the potential of the haptic to elicit important potential for re-enactment, and thus promote simulated actions which form a basis for mathematical reasoning [4]. In contrast, one pair (P9P10) appeared to perceive the feedback as a property of the objects in the game. P10 stated "It feels like I am the ball" and "When there isn't anything in the way it feels like you can go anywhere. When you're inside the box it's like when you go up you can't go anywhere." This was experienced when P9P10 deliberately moved the open cube so that they could not see the ball (cursor) when it was inside the box (fig 6). When P9P10 were asked how they collected the fuel cells, they responded: "we were going like this (showing with their hand that were trying to follow the contour of the base of the cube) and "if we could not make it then we moved it like this" indicating that they moved the haptic back and forth relying on the sensory feedback. Another pair with gaming experience (P17P18) also made movements based on haptic feedback alone, showing awareness of which fuel cells they collected each time. This game play mode is significant for various reasons: a) it was discovered by the children b) the haptic feedback is used as instrument for game play c) there is learning potential to integrating this mode into the game play to foster enactment of the properties of the cube.

\section{Design implications and future work}

This paper presents ongoing work on a prototype that integrates haptic feedback into the exploration of geometrical concepts for young children. Our findings suggest that children adopt different grasp styles of the $\mathrm{HD}$ with the Fist Hold Thumb Click being the most common. The way the HD is held influences the control, angle and rotation of the pen. We need to further investigate how different grips effect players' control of the HD, and the salience of their sensory experience. The findings also showed that for typically developing children, haptic feedback given at the same time as related visual feedback tends to be overlooked. This links to the Colavita visual dominance effect which shows that vision tends to dominate over other perceptual modalities when two sensory stimuli are given at the same time [10]. The game play discovered by the children where visual feedback was limited, enabling conscious use of the haptic feedback and supporting enactment of the properties of 3D shapes, is a significant design implication. A game design that enables interchangeable interplay between the salience of visual and haptic feedback, would allow children to engage with sensory cues that differently contribute their experience of shape, and emphasise key concepts of shape, including the 'hidden corner' in a square. Drawing on observation of children's actions, haptic feedback around 'shape' could also be further integrated in the game by restricting movements e.g. getting children to carry the fuel cells through specific routes (i.e. following the contour of the base cube) in order not to lose points. A key aspect to look at in our ongoing work is the degree to which the use of the haptic device enables re-enaction of the modality specific experience [4], and children's use of relevant simulated actions when talking about 3D shape.

\section{Acknowledgements}

The "weDRAW" project has received funding from the European Union's Horizon 2020 Research and Innovation Programme. Grant Agreement No. 732391 The games discussed here, were developed by Neville 
Rudd \& Rodney McConnell of LearnTPM - Serious game development team.

\section{References}

1. Shaaron Ainsworth. 2008. The Educational Value of Multiple-representations when Learning Complex Scientific Concepts. In Visualization: Theory and Practice in Science Education. Models and Modeling in Science Education, John.K. Gilbert, Miriam Reiner and Mary Nakhleh (eds.). Springer

2. Martha W. Alibali and Mitchell J. Nathan. 2012. Embodiment in Mathematics Teaching and Learning: Evidence From Learners' and Teachers' Gestures. J. Learn. Sci. 21,2(April 2012), 247-286.

3. Benedikte S. Als, Janne J. Jensen, and Mikael B. Skov. 2005. Comparison of think-aloud and constructive interaction in usability testing with children. In Proceedings of the 2005 conference on Interaction design and children, 9-16.

4. Lawrence W. Barsalou. 2008. Grounded Cognition. Annu. Rev. Psychol. 59,1(January 2008), 617-645.

5. Petter Bivall, Shaaron Ainsworth, and Lena A. E. Tibell. 2011. Do haptic representations help complex molecular learning? Sci. Educ. 95, 4 (July 2011), 700-719.

6. Richard Lee Davis, Melisa Orta Martinez, Oliver Schneider, Karon E. MacLean, Allison M. Okamura, and Paulo Blikstein. 2017. The Haptic Bridge: Towards a Theory for Haptic-Supported Learning. In Proceedings of the 2017 Conference on Interaction Design and Children, 51-60.

7. Sam Duffy, Sara Price, Gualtiero Volpe, Paul Marshal, Nadia Berthouze, Giulia Cappagli, Luig Cuturi, Nicolo Balzaroti, David Trainor, and Monica Gori. 2017. WeDRAW: Using Multisensory Serious Games to Explore Concepts in Primary Mathematics. In 13th International Conference on Technology in Mathematics Teaching, 467-470.
8. Beste Güçler, Stephen Hegedus, Ryan Robidoux, and Nicholas Jackiw. 2013. Investigating the

Mathematical Discourse of Young Learners Involved in Multi-Modal Mathematical Investigations: The Case of Haptic Technologies. In Visual Mathematics and Cyberlearning. Mathematics Education in the Digital Era, Dragana Martinovic, Viktor Freiman and Zekeriya Karadag (eds.). Springer, 97-118.

9. Insook Han and John B. Black. 2011. Incorporating haptic feedback in simulation for learning physics. Comput. Educ. 57, 4(December 2011), 2281-2290.

10. David Hecht and Miriam Reiner. 2008. Sensory dominance in combinations of audio, visual and haptic stimuli. Exp. Brain Res.193,(2008),307-314.

11. Carey Jewitt. 2008. Multimodality and Literacy in School Classrooms. Rev. Res. Educ. 32, 1 (February 2008), 241-267.

12. George Lakoff and Rafael Núñez. 2000. Where mathematics comes from: How the embodied mind brings mathematics into being. Basic Books, N.Y.

13. Saija Patomäki, Roope Raisamo, Jouni Salo, Virpi Pasto, and Arto Hippula. 2004. Experiences on haptic interfaces for visually impaired young children. In Proceedings of the 6th international conference on Multimodal interfaces, 281-288.

14. Mandayam A. Srinivasan and Cagatay Basdogan. 1997. Haptics in virtual environments: Taxonomy, research status, and challenges. Comput. Graph. 21, 4 (July 1997), 393-404.

15. Feng Wang and Michael J. Hannafin. 2005. Designbased research and technology-enhanced learning environments. Educ. Technol. Res. Dev. 53, 4 (2005), 5-23.

16. Zacharias C. Zacharia. 2015. Examining whether touch sensory feedback is necessary for science learning through experimentation: A literature review of two different lines of research across K16. Educ. Res. Rev. 16, (October 2015), 116-137. 